\title{
REFLEXIONES FILOSÓFICAS EN TORNO A LOS SISTEMAS DE MEDICIÓN ${ }^{1}$
}

\author{
PHILOSOPHICAL REMARKS ON MEASURING SYSTEMS \\ NYDIA LARA ZAVALA \\ Universidad Nacional Autónoma de México \\ nydialz@yahoo.com
}

\begin{abstract}
RECIBIDO: 05/05/2018
ACEPTADO: $17 / 07 / 2018$
\end{abstract}

Resumen: Pese a que las medidas forman parte de nuestra vida cotidiana y son fundamentales para el desarrollo de la ciencia y la ingeniería, el hecho es que aún tenemos serias dificultades para comprender a qué refieren: ¿A algo que intrínsecamente pertenece al mundo o a una imposición humana arbitrariamente elegida que nos permite medirlo? En el presente trabajo se analizan las dos posturas para ver los pros y contras que cualquiera de las respuestas conlleva. Argumentamos que es menos problemático asumir que nuestras unidades de medida son imposiciones humanas arbitrarias y no magnitudes que pertenecen a la naturaleza. Desde nuestra perspectiva, lo que es menester entender es que nada es medible si no se estipula una unidad de medida y la elección de esa unidad, aunque sea arbitraria, es la que nos va a permitir obtener las medidas del mundo.

Palabras clave: Metrología, Unidad de medida, Valor real de medición, Error, Incertidumbre.

\begin{abstract}
Although measures are part of our daily lives and are fundamental to the development of science and engineering, the fact is that we still have serious difficulties to understand what they refer to: to something that inherently belongs to the world or a human imposition arbitrarily chosen that allows us to measure it? In the present work the two positions are analyzed to explore the pros and cons that any of the answers entails. We argue that it is less problematic to assume that our units of measurement are arbitrary human impositions and not magnitudes that belong to nature. From our perspective, what is necessary to understand is that nothing is measurable unless a unit of measure is stipulated and the choice of that unity, even if arbitrary, is the one that will allow us to obtain the measures of the world.
\end{abstract}

Keywords: Metrology, Unit of measurement, Real value of measurement, Error, Uncertainty.

\section{Introducción}

Un tema sumamente importante, aunque sólo intermitentemente explorado por los filósofos a lo largo de la historia, está relacionado con los instrumentos de medición que muchas veces nosotros utilizamos en la vida cotidiana, pero que indiscutiblemente son fundamentales para el desarrollo del conocimiento de la

1 Investigación realizada gracias al Programa UNAM-DGAPA-PAPIIT clave IN403317 Epistemología de la tecnología y el desarrollo del conocimiento científico. 
ciencia y la ingeniería para obtener muchos de sus resultados. Aunque encontramos importantes reflexiones filosóficas en torno a la medición desde el siglo XIX, lo cierto es que es un tópico que a veces se atiende pero que luego se abandona sin que se logre realmente obtener una comprensión cabal de lo que es la medición y su enorme importancia epistémica y práctica para el mundo moderno.

Esto puede deberse, en parte, al hecho de que su relevancia en el desarrollo del conocimiento científico es realmente muy joven. Su origen lo podemos rastrear más o menos durante los siglos XVII y XVIII, cuando se sentaron las bases metafísicas, ontológicas y epistémicas que desde entonces caracteriza una buena parte de la actividad del trabajo científico, donde la tecnología de la medición adquirió una trascendencia extrema para tratar de entender el no menos complejo e intrincado comportamiento de la naturaleza (Mari, 2003, p. 21). Empero, desde su mismo origen quedó abierta una pregunta que hasta la fecha no hemos sido capaces de contestar: ¿Hay efectivamente aspectos cuantitativos en los objetos, fenómenos y procesos independientemente de su medición o no? (Berka, Measurement, p. 206).

El objetivo de este trabajo es tratar de explorar esta pregunta desde dos vertientes: la primera es la denominada 'realista' que sostiene que el mundo tiene medidas y que nuestros instrumentos de medición sólo nos permiten aproximarnos a su verdadero valor. La segunda es una postura propia, quizá muy cercana pero no idéntica al convencionalismo, donde sostenemos que en el mundo encontramos ciertas características, atributos o propiedades que se pueden medir, pero éstos carecen en sí mismos de medidas. Lo que queremos sostener es que son nuestros instrumentos de medición los que nos ofrecen sus medidas, por lo que la medición directamente dependen de las unidades y sus escalas que creamos como estándares para medir ciertas características, atributos o propiedades del mundo, pero el mundo mide lo que los instrumentos que creamos con esos estándares marcan lo que se mide.

\section{Origen y problemas del realismo}

Muy probablemente por el reconocimiento de que el mundo, tal como lo percibimos, es demasiado complejo, desde el siglo XVII se trató de simplificar dividiendo a la percepción en cualidades primarias y secundarias. Se asumió que sólo las primarias eran reales, mientras que las secundarias se consideraron como meras ficciones de nuestros sentidos sin ningún valor cognitivo real. Galileo, por ejemplo, nos dice: 
...yo juzgo que, si los oídos, las lenguas y las narices se eliminaran, la figura, los números y el movimiento sin duda permanecerían, pero no el olor o el sabor o el sonido, que, sin el animal viviente, no puedo creer que sean otra cosa más que nombres, así como las cosquillas no son otra cosa mas que un nombre cuando la axila o la membrana de la nariz se eliminan (Galileo, Opere, IV, 336, ff.).

Desde entonces se catalogó como lo real, objetivo y verdadero sólo a aquellas cualidades, características o propiedades observadas que fueran susceptibles de ser medidas y cuantificadas. Pero este postulado casi de inmediato provocó severos conflictos para entender si las medidas eran propiedades intrínsecas a la realidad así concebida o eran el resultado mostrado por los instrumentos de medición.

De acuerdo con la teoría que postula la distinción ente cualidades primarias y secundarias (Galileo, Descartes, Boyle y Locke), donde se catalogan a las primarias como aquello que en sí mismo posee propiedades medibles y cuantificables, es obvio que se pensara que las medidas de hecho pertenecen a las cosas que se miden y son independientes de con qué o cómo se miden (Joel Michell, 2004, p. 1). Este es el origen de la corriente filosófica de la medición denominada 'realismo' que, pese a que es ampliamente aceptada hasta la actualidad, conlleva una serie de acertijos metafísicos que a nuestro juicio, enreda el tema a tal extremo que se acaba por no entender no sólo qué es y a qué refiere la medición, sino qué es lo que se pretende lograr cuando medimos las partes del mundo que nos interesa con la cada vez más compleja tecnología de la medición.

Desde la perspectiva de un realista, hay una diferencia entre los valores reales de la parte del mundo que queremos medir y la elección del instrumento con el que lo medimos. Los instrumentos, nos dicen, sólo parecen ofrecernos una aproximación estadística de sus verdaderas medidas, por lo que los avances en la tecnología de la medición, desde su perspectiva, tienen como propósito acortar el sesgo entre las medidas reales y los valores estadísticos que reflejan los instrumentos utilizados para medirlos.

Por el valor estadístico de las medidas experimentales, se asume una premisa de corte metafísico que aún se defiende tanto por muchos científicos, como por metrólogos y filósofos: que en la naturaleza hay medidas reales aunque nuestros instrumentos de medición sólo nos pueden dar aproximaciones de ellas (ver Trout, 2000, pp. 272-276 y Byerly y Lazara, 1973, 10-28. Para una discusión en torno a la postura de los metrólogos, ver Mari y Giordani, 2013, Cap. 4). Esto se traduce en la creencia de que los obstáculos para alcanzar el verdadero conocimiento no es un problema ni de la naturaleza ni de las teorías que dan 
cuenta de ella, sino de los instrumentos de medición que se utilizan, esto es, el problema epistémico se le imputa a la falta de precisión, exactitud y confiabilidad de la tecnología de la medición.

La idea de que las medidas son propiedades que pertenecen al mundo, independientemente de si las medimos o no, aunque está ampliamente difundida, conlleva a tener que enfrentar problemas insolubles. Dentro de ellos podemos destacar el hecho de que a lo único que tenemos acceso son a las medidas que obtenemos con nuestros instrumentos, por lo que declararlos incapaces de brindarnos el valor real de las medidas de las partes del universo que nos interesan, es una forma muy efectiva de negar la posibilidad de saber cuánto efectivamente mide algo, sobre todo si consideramos que se está hablando de una supuesta medida real que abiertamente se reconoce que nosotros ignoramos cuál es. $^{2}$

Lo que es un hecho es que bajo el supuesto de que hay un valor real de medición, independiente de las medidas que se toman con los instrumentos que se utilicen, se tiende a pensar que ningún procedimiento de medición es exacto (Kyburg, 1984, p. 3) por lo que la ilusión de encontrar el supuesto valor real de lo que se mide se vuelve, desde esta perspectiva, un problema imposible de resolver.

Por la aceptación de que no es posible obtener con nuestros instrumentos el valor real de la medida de las cosas del mundo, desde el siglo XIX surge la denominada 'Teoría del error' ampliamente aceptada tanto por metrólogos como por filósofos donde, como lo indica el mismo nombre de la teoría, su función es detectar los errores de medición supuestamente comparados con un hipotético valor real que abiertamente se reconoce que nadie sabe cuál es. Esto es así, porque según esta teoría el error se concibe como la diferencia entre los resultados de una medición y su valor real. Se conocen los resultados de la medición, pero se desconoce el valor real y si se desconoce el valor real, es evidente que también se desconoce el rango de error entre los resultados de la medición y el valor real (X. Ye et al, The new concept of measurement of error theory, Measurement 83, 2016, p 97).

Lo cierto es que la premisa que sostiene que en el universo hay valores reales de medición y la aceptación de que nuestros instrumentos contienen errores que nos impiden llegar a conocerlos, nos ha llenado la cabeza de problemas irresolubles que en la literatura se reflejan de diversas maneras. Un claro ejemplo

\footnotetext{
2 Esta idea queda muy bien expresada por Mari, quien cita el Vocabulario Internacional de Metrología de la ISO DE 1993 que dice: "el verdadero valor de una cantidad es un concepto ideal y, en general, no puede ser conocido exactamente" (Mari, p. 21).
} 
de la clase de dificultades que se discuten sobre este tema, nos lo ofrece Hasok Chang en su libro Inventing temperature.

Chang nos proporciona una tabla en donde compara las discrepancias que se obtienen en las escalas de medición entre tres termómetros cuando los tubos se llenan con mercurio, con alcohol y con agua respectivamente. En los tres casos el $0^{\circ}$ equivale al punto de congelación del agua y el $100^{\circ}$ a su punto de ebullición. Como el rango es muy grande (del 0 al 100) los tres termómetros subdividen su escala en porciones de 10 y cada decena a su vez se subdivide en 10 partes, quedando una escala idéntica en todos ellos. Sin embargo, cuando los tres termómetros se utilizan para medir la misma muestra al mismo tiempo, lo que se obtiene es que cuando, por ejemplo, el termómetro de mercurio marca $50^{\circ}$, el de alcohol marca $44^{\circ}$ y el de agua $26^{\circ}$.

La pregunta en estos casos se ha planteado como sigue: ¿Cómo se elige qué termómetro utilizar para medir la temperatura? Esto es: ¿Cuál de los tres se acerca más a la temperatura real de la muestra medida? El problema es que para poder decidir eso, necesitaríamos contar con un termómetro exacto para que nos proporcione la medida real de la muestra y podamos elegir cuál de los tres instrumentos es el que más se acerca a ella. Pero eso es justo lo que no se tiene, por lo que visto así irremediablemente nos encierra en un círculo vicioso y nos deja ante un problema que por más que le demos vueltas parece no tener solución. K. F. Gauss, uno de los padres de la teoría del error, por ejemplo, afirma: "cuando se analiza el significado de las mediciones que se han obtenido, el experimentador trata de estimar su verdadero valor, el valor que hubiera producido el mejor instrumento realizable" (aparece en Mari, p. 21). Empero, bajo esta perspectiva, no tendríamos ni siquiera los criterios para determinar cuál podría ser el instrumento que se acercara más a los valores reales de lo que medimos.

Lo que es un hecho es que los fundadores de la ciencia moderna de los siglos XVII y XVIII nos dejaron inmersos en el mito de Sísifo al proponer que en el mundo existen medidas reales que nosotros tendríamos que descubrir. El problema se vuelve grave, porque al mismo tiempo se acepta que nuestros instrumentos de medición sólo nos dan valores inexactos de la realidad que se mide. Bajo esta perspectiva parece que la labor del metrólogo se convierte en encontrar la manera de mejorar la instrumentación hasta lograr un objetivo: acercar cada vez más la medida que refleja el instrumento al valor real de la muestra, ilusión que, como bien lo ilustra Chang, es humanamente inasequible.

No se está afirmando que no puede haber errores de medición, pero de ahí a suponer que todas las medidas son, por definición, inexactas o erráticas parece ser una exageración sin más sustento que una asunción metafísica más que 
cuestionable. Lo que sostenemos es que puede haber y, de hecho las hay, discrepancias de medición sobre todo si se considera que al medir es necesario tomar en cuenta la estabilidad de lo que se quiere medir, la estabilidad del instrumento de medición, la apreciación del operador que ejecuta la medida y los factores del universo que pueden influir en la toma de esa medida. Por todos estos factores en la ciencia, en el desarrollo tecnológico y en la industria, las medidas que requieren de mucha precisión exigen la utilización de instrumentos calibrados conforme a los patrones de referencia por instituciones certificadas en metrología para comprobar que las escalas se ajusten a los valores correspondientes a las unidades de medida aceptadas. Asimismo, se requieren operadores bien entrenados para tomar las medidas de manera adecuada, lo cual exige que las medidas se realicen varias veces sobre la misma muestra y que se especifiquen los valores de influencia que puede ejercer el medio ambiente tanto en el instrumento de medición como en la muestra que se mide. Pero también se requiere calcular la incertidumbre de las medidas obtenidas por reiteración, que no siempre son iguales, pues para un operador el valor de una medida puede ser, digamos, $7.00 \mathrm{~cm}$, para otro 6.99, para otro 7.01 y aún para otro 6.98. Empero, sostener que las discrepancias son errores involucra suponer que de alguna manera se tiene conocimiento de lo que es correcto, cosa que no existe en el mundo de la medición. Lo cierto es que la variedad de medidas que se obtienen con un mismo instrumento sobre una misma muestra, en general se debe a que las marcas de las escalas tienen diversos espacios y grosores, por lo que un mismo operador, o varios, pueden interpretar de manera diferente qué medida marca la escala. Pero a pesar de las diferencias (que en general son muy pequeñas), la diversidad no se puede considerar como errores de medición, sino como diferencias de apreciación, que es realmente lo que en metrología ahora se denomina 'incertidumbre'. ${ }^{3}$

De hecho, nuestros instrumentos de medición se han ido mejorado y poco a poco se han logrado institucionalizar, no porque estemos alcanzando el conocimiento de las medidas reales del mundo, sino porque cada día las unidades que marcan los estándares que nos van a servir para medir se hacen más estables, que es el trabajo que realmente les compete llevar a cabo a los metrólogos como lo veremos más adelante.

\footnotetext{
3 En la literatura tanto filosófica como metrológica se manejan dos sentidos del término 'incertidumbre': el que se acuña de la teoría del error, donde se proponen valores ideales de medición y el que propone el JCGM 200:2012, International Vocabulary of Metrology - Basic and general concepts and associated terms (VIM), 3rd Edition, que trata de corregir la versión tradicional con una acepción semejante a la que estamos empleando nosotros. De todas maneras, este es un tema que sin duda requiere de un análisis conceptual mucho más puntual para desentrañar sus ambigüedades.
} 
Lo que basta reconocer, para liberarnos de premisas metafísicas desorientadoras, es que el punto medular para abordar el tema de la medición no puede partir de la idea de que el mundo posee medidas, sino que tiene que ver con la necesidad de entender por qué ningún sistema de medición sería posible sin la especificación de lo que se va a considerar una unidad de medida. Revisemos con detalle este tema para apreciar su importancia.

\section{Unidad de medida}

Lo que queremos sostener a continuación es que la fuente del conocimiento de la medición está fincada en su unidad de medida. Por eso, cualquier sistema de medición requiere de inicio estipular una unidad, idealmente invariable, que nos sirva como patrón de referencia para empezar a contar cuántas veces cabe esa unidad en lo que queremos medir. Esto es, la elección de esa unidad es la que sirve de estándar o patrón para calcular una magnitud, entendiendo por 'magnitud' la medida de algo conforme a la unidad elegida. Esto es importante considerarlo, porque muchas cosas diferentes comparten una o varias propiedades medibles en común. Por lo mismo, se pueden medir muchas cosas diferentes utilizando la misma unidad de medida que nos sirve para determinar la magnitud de la característica, atributo o propiedad a la que se aplica esa unidad. Esto significa que sin la especificación de lo que se va a considerar nuestra unidad de medida, nada sería medible. La razón es que el mundo mide lo que nuestras unidades de medición indican que mide.

El problema es que la elección de una unidad de medida, si no hay consensos sociales, puede ser totalmente arbitraria. Esto es, si se requiere medir, digamos, el largo de un mueble, se puede utilizar como unidad de medida el tamaño de la cuarta de una mano, el tamaño de un palo o el tamaño de un listón. Con esto lo que se quiere enfatizar, por lo pronto, es que mientras que el tamaño del mueble sin duda alguna es una propiedad del objeto, su medida, aunque posible, no lo es. Lo mismo se puede decir de la cuarta de la mano, del palo o del listón que en cierto momento pueden servir como unidad de medida. Los tres poseen tamaño y con cualquiera de los tres se puede medir el largo del mueble, pero en sí mismos ellos no poseen medida. No obstante, la medida que se puede obtener del largo del mueble va a depender de la unidad de medición empleada, porque cualquiera que se elija va a fungir como el patrón de referencia que nos sirve para iniciar el proceso de medición. Pero por supuesto que la medida cambia y varía dependiendo de qué unidad de medición se emplee, esto es, la medida del mueble variará en relación a la unidad de medida utilizada (la cuarta 
de la mano, del palo o del listón). La elección de esa unidad, como se dijo anteriormente, se puede elegir de manera completamente arbitraria, es decir, la fijamos nosotros y la parte del mundo que medimos se adapta a ella. Pero es evidente que distintas cuartas de distintas manos, distintos palos o distintos listones pueden tener tremendas variaciones $\mathrm{y}$, por lo mismo, no nos van a ofrecer los mismos resultados de medición cuando los comparamos. ${ }^{4}$

De hecho, lo que motivó a los intelectuales revolucionarios franceses durante el siglo XVIII a buscar un consenso social con el metro, fue, en primer lugar, minar la arbitrariedad de la nobleza y el comercio que libremente cambiaba sus unidades de medida incrementándolas o disminuyéndolas a su conveniencia y, en segundo lugar, para establecer una unidad de medida "objetiva" y fundamentalmente estable, que permitiera derivar las otras, como el gramo o el litro, con la intención de que pudieran ser utilizadas como patrones de referencia por cualquiera y que permitiera la colaboración entre diversas personas independientemente de su credo, ideología, procedencia o clase social (ver Mari, p. 21).

Pero el metro no fue pensado como una unidad de medida arbitraria. Sus creadores, embebidos en el espíritu cientificista de su época, partieron de la idea de que el mundo tiene medidas y con esta convicción decidieron crear una unidad que procediera de las medidas de la misma Tierra con la intensión de que fuera universal y accesible para cualquiera. En 1791 se propuso que el metro fuera la diezmillonésima parte de la distancia entre el Polo Norte y el Ecuador pasando por el meridiano de París. El problema evidente era, primero, cómo medir esa distancia sin recurrir a una unidad de medida para hacerlo, ya que hablar de la diezmillonésima parte de un cuadrante de la Tierra no es una medida, sino una proporción geométrica abstracta y, segundo, que la distancia propuesta para calcular la diezmillonésima parte de ese cuadrante era excesiva.

Como es imposible tomar una medida sin contar con una unidad para hacerla se recurrió a la toesa ${ }^{5}$ y al círculo de reflexión de Borda para obtener las medidas angulares. Esta elección se debió a que la toesa era una barra ampliamente utilizada en París y el círculo de Borda se consideraba un confiable instrumento de medición para hacer operaciones geodésicas. Para enfrentar el segundo problema, se optó por sólo medir la parte que iba de Dunquenque, Francia, a

\footnotetext{
${ }^{4}$ Recuérdese el ejemplo de los termómetros de Chang.

${ }^{5}$ Se sabe que se utilizó la toesa, pero había varias. Hay quienes dicen que la que se empleó fue la del Perú, otros dicen que la castellana y aún otros que la de Burgos que se utilizaba en París. Adler en su libro La medida de todas las cosas, dice que se utilizaron cuatro varas de platino, cada una de dos toesas de longitud, que equivaldría aproximadamente a $3.898 \mathrm{~m}$, donde cada toesa tendría una longitud aproximada de $1.949 \mathrm{~m}$.
} 
Montjuic en Barcelona, España. Dos científicos, Méchain, astrónomo y geógrafo, y Delambre, astrónomo y matemático, fueron los encargados de tomar esas medidas. Con los resultados obtenidos se procedió a hacer el cálculo matemático para obtener la medida entre el Polo Norte y el Ecuador, para después calcular la diezmillonésima porción de esa distancia con la finalidad de determinar el tamaño del metro.

En su momento el metro se concibió como la primera unidad de medida obtenida de la misma naturaleza, lo cual para ellos significaba la garantía de su neutralidad, objetividad y validez universal. Pero, además, la magnitud de lo que debería medir un metro estaba previamente estipulada en abstracto antes de que se obtuvieran los resultados de medición de lo que se determinó que debería de ser un metro (la diezmillonésima parte del cuadrante del Polo Norte al Ecuador).

Lo que es digno de llamar la atención es que este caso parece que representa la primera ocasión conocida donde se determina una unidad de medida con base en un cálculo matemático extraído de mediciones parciales obtenidas con otras unidades de medida (la toesa y el círculo de Borda). Lo cual quiere decir que, aunque ya nadie utiliza toesas y círculos de Borda para hacer sus mediciones, el metro nació con el estatus de longitud gracias a esas unidades de medida. Sin embargo, todos sabemos que pese al titánico esfuerzo de medición que llevaron a cabo Méchain y Delembre, lo cierto que el metro, si bien nació de un cálculo matemático para estipular su medida, no mide la diezmillonésima parte de la distancia entre el Polo Norte y el Ecuador, sino la cantidad que estipularon los dos científicos involucrados en el proceso para determinar qué era un metro. Empero, eso de ninguna manera ha obstaculizado el hecho de que el metro se haya convertido en el patrón estándar más aceptado hasta la fecha y que junto con las otras unidades que se derivan directamente de él, como el kilo y el litro, sean las unidades que conforman el denominado 'sistema métrico decimal', que representan las unidades de medida más utilizadas en el mundo, con la inmensa ventaja que acarrea el acuerdo social que eso conlleva.

No obstante, y pese al halo cientificista y democrático que lo envuelve, el sistema métrico decimal no ha sido adoptado por todos. Estados Unidos, Birmania y Liberia, hasta la fecha utilizan otras unidades de medidas distintas a las del sistema métrico decimal. Esto es un problema, pues el hecho de que aún no tengamos acuerdos universales en torno a las unidades de medidas que utilizamos, puede ocasionar confusiones y desastres irreparables. Como ejemplo tenemos el caso del Orbitador del Clima de Marte (Mars Climate Orbiter), donde la compañía Lockheed Martin Astronautics, que se encargó de su diseño y construcción, realizaba sus cálculos con el sistema anglosajón de medidas (pies, libras, millas) y el Jet Propultion Laboratory, responsable de programar los 
sistemas de navegación del Orbitador los realizaba con el SI (metros, kilogramos, kilómetros). Por un lamentable error de comunicación, ninguna de las dos instituciones involucradas especificó sus unidades de medida por lo que la órbita del dispositivo se calculó mal y se presume que se destruyó porque hizo contacto con la atmósfera de Marte.

Con este ejemplo lo que se quiere enfatizar es que a pesar de que la elección de las unidades de medición tiene este carácter arbitrario y, por lo mismo, cualquiera puede elegir la que mejor le parezca, el trabajo de equipo exige acuerdos y consensos sociales sobre cuál elegir. La moraleja de experiencias de esta clase es que cuando hay un proyecto común que requiere de medidas, los integrantes tienen que estipular qué sistema de medición van a utilizar pues el mundo mide lo que nuestras unidades de medida dicen que mide. Si se entiende el punto, es fácil darse cuenta de que el fracaso del Orbitador de Marte propiamente hablando no se puede entender como un problema de error de medición. Dado el prestigio de las dos instituciones involucradas, podemos asumir que las dos realizaron sus medidas de la manera más precisa posible, pero no con las mismas unidades de medida. El problema entonces no fue la medición que cada institución obtuvo por su cuenta, sino el no considerar que dos unidades de medida diferentes estaban socialmente aceptadas y, por lo mismo, que era posible obtener datos y resultados correctos pero distintos al medir los mismos hechos del mundo con sistemas de medición distintos.

Lo que siempre se tiene que considerar es que el mundo en sí mismo no tiene medidas sino que las medidas se obtienen a partir de nuestras unidades de medición. Es decir, el mundo no mide lo mismo cuando se utiliza, digamos, el sistema anglosajón o el sistema métrico decimal. Los dos en sí mismos pueden brindarnos medidas muy precisas del mundo, pero los resultados son distintos simple y sencillamente porque las unidades de medidas son distintas. Si se reconoce este hecho, podemos aseverar que el problema de fondo es social. La falta de acuerdos internacionales sobre qué unidades de medidas vamos a utilizar hacen que lo que medimos del mundo no se pueda unificar y, aunque se supone que hay equivalencias que nos permiten convertir los resultados de una unidad a otra, por experiencia sabemos que ninguna equivalencia es exacta (Yoder y Morley, 2016, p.15) ${ }^{6}$. Si no se requiere mucha precisión, esto quizá no representa

\footnotetext{
${ }^{6}$ Parte de la razón, aunque no la única, es que el sistema anglosajón es mucho más antiguo que el sistema métrico decimal y eso hace que no sea ni fácil ni coherente establecer sus equivalencias. La base del sistema anglosajón es el 12 y no el 10 como en el decimal, por lo que incluso las equivalencias internas al mismo sistema anglosajón son difíciles de manejar. Las conversiones de un sistema a otro, al no tener la misma base, complica muchísimo la posibilidad de tener valores equivalentes confiables. Además, se tiene que considerar que en las dos unidades comparadas a su
} 
un gran problema; sin embargo, si se necesita, como en general es el caso de muchos de los desarrollos de la ciencia y la ingeniería, lo más conveniente es utilizar la misma unidad para hacer las mediciones que se requieran para que el mundo mida aproximadamente lo mismo independientemente de quién o dónde se mida.

El hecho es, y para resumir lo que hasta aquí se ha dicho, que la estipulación de una unidad de medida es una condición necesaria para medir. Empero, su elección se puede hacer de manera personal, grupal o institucional. Por razones obvias y evidentemente prácticas, lo más deseable es que nuestras unidades de medidas sean institucionales, para que todos usemos las mismas y que con ellas podamos obtener, en principio, los mismos resultados de medición realizados por distintos agentes y en distintos lugares, con el fin de poder comunicar, compartir e intercambiar con los demás esos resultados de manera confiable. Ese es el papel que actualmente tiene, por ejemplo, el Sistema Internacional de Unidades (conocido como el SI) que se utiliza actualmente en una muy buena parte del mundo, donde está legalmente acordado emplear sus unidades de medida de manera oficial. Así tenemos, por ejemplo, el metro para medir longitudes, el kilo para medir masas, el segundo para medir el tiempo, el amperio para medir corrientes eléctricas, el kelvin para medir la temperatura termodinámica, el mol para medir la cantidad de sustancias o la candela para medir la intensidad luminosa. Estas siete unidades son consideradas las básicas y prácticamente todas las demás se derivan de ellas de manera coherente.

Con todo, incluso utilizando las mismas unidades de medidas, el tema de la precisión es una preocupación constante de la metrología, que es la disciplina que se dedica al estudio, pruebas, calibración, mantenimiento y mejoramiento de los instrumentos de medición para garantizar la estabilidad de nuestras unidades de medidas.

La precisión directamente depende de la estabilidad de nuestras unidades de medidas, la cual está íntimamente relacionada con la calibración de los instrumentos que se utilizan para realizarlas (reglas, balanzas, relojes, termómetros, osciloscopios, etc.). Como es otro tema central para entender la medición, a continuación pasaremos a explicar en qué consiste la estabilidad y cuáles son los problemas epistémicos que enfrenta y cómo se han tratado de solucionar.

vez existen incertidumbres internas, por lo que las equivalencias siempre son problemáticas y requieren de muchos ajustes. 


\section{Estabilidad de los instrumentos de medición}

Vale la pena aclarar que la confianza o desconfianza de los resultados de nuestra medición, no recae, como sostiene la tradición realista, en las características o propiedades de los objetos, eventos o fenómenos que se miden, sino en la estabilidad o inestabilidad de nuestras unidades de medida. No obstante, desde el siglo XVII se ha popularizado la idea de que el mundo tiene medidas y que nuestros instrumentos de medición sólo nos pueden proporcionar medidas inexactas de su verdadera realidad. Esto ha propiciado que los estudiosos de la medición pasen por alto el hecho de que las unidades de medida no son y no pueden ser entidades abstractas (como la diezmillonésima parte del cuadrante de la Tierra del Polo Norte al Ecuador), sino realizaciones físicas concretas que sirvan como patrones o estándares de referencia objetivos para calibrar el resto de los instrumentos de medición que se van a utilizar para obtener resultados equivalentes de medición independientemente de quién o dónde se tomen.

Pero es un hecho indiscutible que la realización física de cualquier unidad de medida puede sufrir fluctuaciones, con el problema de que si el patrón varía, los instrumentos que se basan en él también variarían. Por esta razón hay quienes sostienen que parte de la labor de la metrología consiste en tratar de encontrar los valores reales de la naturaleza para corregir los errores de los patrones físicos de las unidades de medición (Mari y Giordani, 2013). El problema que esta visión encierra es doble, pues, por un lado, esas supuestas correcciones a los patrones físicos, en general, son modelos teóricos que refieren a medidas que no se han realizado o que, si se realizaron, hubieran tenido que hacerse a través de algún instrumento de medición existente, con el inconveniente adicional de que para esta postura serían medidas aproximadas o erráticas, por lo que desde esta línea de pensamiento no hay posibilidad de salir de su círculo vicioso (ver Eran Tal, 2016). Pero, por el otro lado, la mera suposición de que la metrología constantemente esté cambiando los valores de nuestras unidades de medida para ajustarlas a las verdaderas medidas de la naturaleza, implicaría algo aún más dramático que un problema meramente conceptual: que no habría estándares fijos para determinar la pertinencia de los resultados de nuestras medidas.

Sin embargo, basta recordar para desechar esta versión, que actualmente existen patrones de medidas muchísimo más exactas que las que obtuvieron, digamos, Méchain y Dalambre cuando hicieron sus cálculos para determinar la diezmillonésima parte del cuadrante terrestre. Pero sería absurdo modificar el metro que ellos obtuvieron en función de nuevos hallazgos, pues la institucionalización de una unidad de medida implica un esfuerzo brutal para habituar a la sociedad a utilizarla. De hecho, es tan complicado imponer una 
unidad universal de medida, que aún después de un poco más de dos siglos, el sistema métrico decimal todavía no puede ser implantado en todo el mundo. Imaginemos, pues, que si con cada nueva medición de la naturaleza, procediéramos a modificar nuestras unidades de medición, el mundo de la medición sería un verdadero caos y muy posiblemente estaríamos en una situación muy parecida a la que prevalecía antes de que se instituyera el sistema métrico decimal como el más aceptado y usado por la sociedad actual.

Afortunadamente la verdadera historia es otra: la metrología tiene objetivos prácticos, aunado a la imperiosa necesidad de que los patrones institucionalizados sirvan para que sean fácilmente reproducibles para que todos compartamos los mismos resultados de medición. Con esto en mente, el metrólogo trata de elegir los materiales más estables posibles y tener en las mejores y más cuidadosas condiciones los patrones de medición para evitar en lo posible que sufran modificaciones. Por esta razón, la metrología requiere de mucha investigación y los patrones se van perfeccionando a lo largo de su historia conforme nuevos y más estables materiales se consiguen o se perfeccionan los instrumentos patrón. Pero la unidad de medida, una vez que está socialmente aceptada e institucionalizada, no se cambia, sólo la estabilizan más para que sufra el menor número de fluctuaciones posible.

De hecho, basta recordar que la primera representación física del metro se hizo de platino porque se consideró que era un material muy confiable. A esa barra se le llama 'el metro de los archivos'. Con el tiempo y, a pesar de los cuidados que se tenían, los metrólogos detectaron que el patrón del metro no era tan invariable como lo habían supuesto. Por eso' decidieron hacer una aleación de $90 \%$ de platino con un $10 \%$ de iridio para hacer un nuevo patrón, que aunque se basó en el de los archivos, se hacía mucho más fuerte y confiable para evitar deformaciones y alteraciones cuando se usaba para la calibración. También le dieron una forma denominada 'sección Tresca' (como si fuera una especie de $\mathrm{X}$ gruesa) para darle mayor estabilidad. Este metro patrón estuvo en uso hasta 1960. Empero, nuevos estudios pudieron detectar que la barra tenía mínimas fluctuaciones que podían superarse utilizando la longitud de onda en el vacío de la línea rojo-naranja del elemento kriptón 86 , que se supone proporciona una medida patrón del metro mucho más estable que la barra de platino e iridio.

Cabe aclarar que todos estos ajustes al metro se han hecho mejorando la estabilidad del de los archivos, a pesar de que se sabe, como se mencionó arriba, que los resultados de Méchain y Dalambre, que fueron los que definieron lo que era el metro, no corresponde exactamente con la diezmillonésima parte del meridiano que va del Polo Norte al Ecuador. Ahora nos dicen que desde 1983 el metro no es la medida del cuadrante de la Tierra, sino la distancia recorrida por 
la luz en el vacío durante 1/299’ 792,458 de segundo. La fantasía de los realistas supone que con esta nueva definición, volvimos a ubicar el metro como una medida natural, pero esto evidentemente es un enredo conceptual, pues, en primer lugar, esa cantidad refiere a una medida evidentemente realizada con unidades aceptadas y, en segundo lugar, lo que se hizo en realidad fue tomar como referente el patrón más estable del metro y sacar las medidas de lo que tardaba la luz en recorrerlo. Pero el metro patrón, nos guste o no, es una unidad de medida muy utilizada en la actualidad, pero no es natural. Ahora bien, si esta nueva definición del metro es práctica o no, la historia nos lo dirá. Lo que es un hecho incontrovertible es que nadie ha tirado sus réplicas del metro calibrado con el anterior patrón, porque a los metrólogos se les ocurrió imponer una nueva definición de lo que consideran que es un metro. Eso lo tenemos estipulado desde que se aceptó el concepto de metro como nuestra media oficial de la longitud y esperemos que nuestras unidades de medidas se conserven institucionalmente durante mucho tiempo y, aún más, que la parte del mundo que aún no se integra al SI, poco a poco se decida a hacerlo para evitar catástofres del tipo del Orbitador de Marte.

\section{Conclusiones}

Hay sin duda muchos otros temas y problemas relacionados con la medición que son dignos de reflexiones filosóficas, sobre todo cuando caemos en la cuenta de que todavía es necesario despejarlo de premisas metafísicas que sólo han contribuído a desorientar a los estudiosos y profesionales de la medición planteando acertijos sin solución a un tema que de por sí es sumamente complejo.

En este pequeño ensayo nos dedicamos a criticar la postura realista que sostiene que el mundo tiene medidas. Idea que, como vimos, conlleva la creencia de que es competencia del metrólogo descubrirlas y ajustar poco a poco sus instrumentos de medición hasta supuestamente lograrlo. Pero la misma metafísca que promueve esta creencia, reconoce que esa es una empresa imposible, por lo que de aquí no salen más que enredos y complicaciones que no se pueden resolver. Por eso en este trabajo se optó por partir de la premisa contraria, es decir, asumimos que el mundo carece de medidas y que la única herramienta real con la que contamos para medirlo refiere a nuestras unidades de medida.

Las ventajas que obtenemos desde esta perspectiva es que en nuestro caso, por ejemplo, no aparecen enigmas irresolubles y a nuestro juicio queda mucho 
más claro el recuento histórico de cómo se gestó la necesidad de proponer un sistema universal de medidas y por qué. También nos parece que queda mucho mejor explicada la labor del metrólogo, cuya tarea es encontrar la manera de hacer más estables y confiables nuestras unidades de medidas y no tratar de encontrar medidas que de origen se asumen como misteriosas e inalcanzables. Desde nuestro enfoque no aparece la aberrante idea de que todas nuestras medidas son erráticas e inexactas. Con nuestra posición esa idea queda descartada y se explica mucho mejor el concepto de incertidumbre como una cuestión que tiene que ver con la estabilidad de nuestros patrones o con pequeñas discrepancias relacionadas con la apreciación del operador, pero de ninguna manera como un error intrínseco a las medidas que nos proporcionan nuestros instrumentos.

Reconocemos que queda abierto el problema de que las unidades de medida se eligen de manera arbitraria. La otra postura zanja esta cuestión bajo la asunción de que en el mundo existen las verdaderas medidas y que las unidades que proponemos tienen como fundamento una medida natural que las hace objetivas, neutras y universales de suyo. Nosotros, empero, nos podemos defender en contra de esta creencia, sosteniendo que tenemos como testigo ocular a la historia, donde se nos demuestra que las unidades que en algún momento se proponen como naturales, en realidad son obtenidas de mediciones que no corresponden a una realidad ajena a nuestras propias unidades de medidas. En efecto, conforme nuestras unidades se estabilizan más, nos damos cuenta que aquello que se propuso como una medida real y natural, resulta que no corresponde con la dimensión que generó el patrón que aceptamos e institucionalizamos y, pese a que no son ni reales ni naturales, son las que utilizamos y nos sirven muy exitosamente tanto en la vida cotidiana como en el desarrollo de la ciencia y la ingeniería.

En conclusión, nuestra propuesta parece ser más clara, explicativa y sensata que la realista, con la inmensa ventaja de que desde nuestra perspectiva se evita que aparezcan quimeras y enigmas irresolubles. Es por eso que pensamos que lo que defendemos en este trabajo es una posición más sana y más apta que marca el principio para tratar de comprender y desenredar la serie de temas y problemas inmersos en el estudio de la medición que, hasta la fecha, se nos ha presentado como un estudio elusivo y prácticamente intratable. 


\section{Bibliografía}

ADLER, Ken, The Measure of All Things: The Seven-Year Odyssey and Hidden Error that Transformed the Word, Free Press, New York, 2002

BERKA, Karel, Measurement: Its conceps, theories and problems, D. Reidel Publisher Company, Boston Studies in Philosophy of Science, V. 72, Holanda, 1983

BIMP, "International vocabulary of metrology: Basic and general concepts and associated terms", Copyright of this document is shared jointly by the JCGM member organizations (BIPM, IEC, IFCC, ILAC, ISO, IUPAC, IUPAP and OIML), 3a ed., 2012

Byerly, H.C. and V.A. Lazara, "Realist Foundations of Measurement", Philosophy of Science, 40(1), 1973, 10-28

CAMPBELL, N.R., Physics: the Elements, Cambridge University Press, London, 1920

CHANG, Hasok, Inventing temperature: Measurement and Scientific Progress, Oxford University Press, New York, 2004

DÍEZ, J.A., "History of measurement theory", History and Philosophy of Science and Technology, Vol. III, EOLSS Publishers Co. Ltd, Oxford, 2010

FAN, Huaan, Theory of Errors and Least Squares Adjustment, Universitetsservice AB, Stockholm, 2010

GALILEI, Galileo, Le Opere, IV, Ed. Nazionale, Firenza, 1894

General principles and definitions, International Organization for Standardization, 1998

HELMHOLTZ, Herman, Counting \& Measuring, Van Nostrand, New York, 1930

ISO 5725-1, Accuracy (trueness and precision) of measurement methods and results - Part 1: General principles and definitions, International Organization for Standardization, 1998

JCGM 100:2008, Evaluation of measurement data - Guide to the expression of uncertainty in measurement, (GUM, originally published in 1993), Joint Committee for Guides in Metrology, 2008

JCGM 101:2008, Evaluation of measurement data - Supplement 1 to the "Guide to the expression of uncertainty in measurement" - Propagation of distributions using a Monte Carlo method, (http://www.bipm.org/en/publications/guides/gum.html).

JCGM 104:2009, Evaluation of measurement data - An introduction to the 'Guide to the expression of uncertainty in measurement' and related documents, Joint Committee for Guides in Metrology, 2009 
JCGM 200:2012, International Vocabulary of Metrology - Basic and general concepts and associated terms (VIM), 3rd Edition (2008 version with minor corrections), Joint Committee for Guides in Metrology, 2012, (http://www.bipm.org/en/publications/guides/vim.html).

KYBURG, Henry E. Jr., Theory of Measurement, Cambridge University Press, New York, 1984

MARI, Lucas, “Epistemology of measurement”, Measurement 34 (2003) 17-30, www.elsevier.com/ locate /measurement

Mari, L. and A. Giordani, "Modeling measurement: error and uncertainty", in Error and Uncertainty in Scientific Practice, M. Boumans, G. Hon, and A. Petersen (eds.), Ch. 4, 2013

MICHELL, Joel, "History and Philosphy of Measurement: A realist view", 10th IMEKO TC 7 International Symposium, June 30-July 2, Rusia, 2004

RADDER, Hans, The Philosophy of Scientific Experimentation, University of Pittsburg Press, USA, 2003

RABINOVICH, Semyong, Measurement Errors and Uncertanities Theory and Practice, Springer, N.J., 2005

STEVENS, S.S., "Mathematics, Measurement, Psychophysics", The Handbook of Experimental Psychology, Wiley \& Sons, New York, pp. 1-49, 1951

SUPPES, Patrick y ZINNES, Joseph L., "Basic Measurement Theory", Technical Report No. 45, Institute for Mathematical Studies in the Social Sciences, Applied Mathematics and Statistics Laboratories, Stanford Univesity, Stanford, California, March 15, 1962

TAL, Eran, "Making Time", Brit. J. Phil. Sci., No.67, p. 297-335, 2016

The Epistemology of Measurement: A Model-Based Account, Graduate Department of Philosophy, University of Toronto, 2012

"Old and New Problems in Philosophy of Measurement", Philosophy Compass 8/12, p. 1159-1173, 2013

"Measurement in Science", Stanford Encyclopedia of Philosophy, First published Mon Jun 15, 2015,

https://plato.stanford.edu/entries/measurement-science/

TROUT, J.D., "Measurement", en A Companion to the Philosophy of Science, W.H. Newton-Smith (ed.), Blackwell Publisher, Malden, MA, 2000, pp. 265276.

YE, X., Xiao, X. y Shi, J., The new concept of measurement of error theory, Measurement, V. 83, p 96-105, April 2016

YODER, Jesse y MORLEY, Dick, The Tao of Measurement: A Philosophical View of Flow and Sensors, International Society of Automation, USA, 2016 
